\title{
The Integrals of Entwining Structure
}

\author{
Yuzhuo Yuan ${ }^{1,2}$, Lihong Dong ${ }^{1}$, Zhengming Jiao $^{{ }^{*}}$ \\ ${ }^{1}$ Department of Mathematics, Henan Normal University, Xinxiang, China \\ ${ }^{2}$ Department of Mathematics, Nanyang Normal University, Nanyang, China \\ Email: ${ }^{*}$ zmjiao@htu.cn
}

Received January 31, 2013; revised March 18, 2013; accepted April 27, 2013

Copyright (C) 2013 Yuzhuo Yuan et al. This is an open access article distributed under the Creative Commons Attribution License, which permits unrestricted use, distribution, and reproduction in any medium, provided the original work is properly cited.

\begin{abstract}
In this paper the integrals of entwining structure $(A, C, \psi)$ are discussed, where $A$ is a $k$-algebra, $C$ a $k$-coalgebra and $\psi: C \otimes A \rightarrow A \otimes C$ a $k$-linear map. We prove that there exists a normalized integral $\gamma: C \rightarrow \operatorname{Hom}(C, A)$ of $(A, C, \psi)$ if and only if any representation of $(A, C, \psi)$ is injective in a functorial way as a corepresentation of $C$.
\end{abstract} We give the dual results as well.

Keywords: Entwining Structure; Integral; Forgetful Functor; Natural Transformation

\section{Introduction}

The integrals for Hopf algebras were introduced by Larson and Sweedler $[1,2]$. Integrals have proven to be essential instruments in constructing invariants of surgically presented 3-manifolds or 3-dimensional topological quantum field theories [3-5]. The aim of this paper is to show that some results of recent paper [6] concerning integrals and its properties for Doi-Koppinen structure hold for the more general concept known as entwining structure $[7,8]$. It is a structure of an algebra, a coalgebra and a k-linear map such that several compatibility conditions are satisfied. Unlike Doi-Koppinen structure, there is no need for a background bialgebra, which is an indispensable part of the Doi-Koppinen construction. The bialgebra-free formulation also has a remarkable self-duality property, which essentially implies that for every statement involving coalgebra structure of an entwining structure there is a corresponding statement involving its algebra structure.

This paper is organized as follows. In Section 2, we recall definitions and give examples of entwining structures and entwined modules. In Section 3, we introduce the integrals of entwining structure and analyse its properties generalizing the results of [6]. Finally, in Section 4 we derive the dual form of the integrals of entwining structure and its properties.

\section{Preliminaries}

Throughout this paper, $k$ will be a field. Unless spe-

*Corresponding author. cified otherwise, all modules, algebras, coalgebras, bialgebras, tensor products and homomorphisms are over $k$. For a $k$-algebra $A, \mathcal{M}_{A}$ (resp. $\left.{ }_{A} \mathcal{M}\right)$ will be the category of right(resp. left) $A$-modules and $A$-linear maps. $H$ will be a Hopf algebra over $k$. We omit Sweedler's sigma-notion [9] extensively. For example, if $(C, \Delta)$ is a coalgebra, then for all $c \in C$ we write $\Delta(c)=c_{(1)} \otimes c_{(2)}$.

Definition 2.1 An entwining structure on $k$ consists of a triple $(A, C, \psi)$, where $A$ is a $k$-algebra, $C$ a $k$-coalgebra and $\psi: C \otimes A \rightarrow A \otimes C$, a $k$-linear map satisfying the relations

$$
\begin{gathered}
(a b)_{\psi} \otimes c^{\psi}=a_{\psi} b_{\Psi} \otimes c^{\psi \Psi}, \\
\left(1_{A}\right)_{\psi} \otimes c^{\psi}=1_{A} \otimes c, \\
a_{\psi} \otimes \Delta_{C}\left(c^{\psi}\right)=a_{\psi \Psi} \otimes c_{(1)}^{\Psi} \otimes c_{(2)}^{\psi}, \\
\epsilon_{C}\left(c^{\psi}\right) a_{\psi}=\epsilon_{C}(c) a
\end{gathered}
$$

for all $a, b \in A, c \in C$, where

$$
\psi(c \otimes a)=a_{\psi} \otimes c^{\psi}=a_{\Psi} \otimes c^{\Psi} .
$$

Remark 2.2 Generally, we call the entwining structure in Definition 2.1 a right-right entwining structure. Unless specified otherwise, all the entwining structures mentioned in this paper are right-right entwining structures.

Definition 2.3 Let $(A, C, \psi)$ and $(\tilde{A}, \tilde{C}, \tilde{\psi})$ be two entwining structures, $f: A \rightarrow \tilde{A}$ be an algebra map and $g: C \rightarrow \tilde{C}$ be a coalgebra map. We call 
$(f, g):(A, C, \psi) \rightarrow(\tilde{A}, \tilde{C}, \tilde{\psi})$ is an entwining map if $(f \otimes g) \circ \psi=\tilde{\psi} \circ(g \otimes f)$.

Example 2.4 Let $H$ be a bialgebra, $A$ a right $H$ comodule algebra, $C$ a right $H$-module coalgebra. Then Doi-Koppinen structure $(H, A, C)[10]$ is an entwining structure. The entwining structure map is

$$
\psi: C \otimes A \rightarrow A \otimes C, c \otimes a \rightarrow a_{\langle 0\rangle} \otimes c \cdot a_{\langle 1\rangle} .
$$

If $H$ has a bijective antipode $S$, the $\psi$ is bijective with

$$
\psi^{-1}: a \otimes c \rightarrow c \cdot S^{-1}\left(a_{\langle 1\rangle}\right) \otimes a_{\langle 0\rangle} .
$$

Example 2.5 [3] Let $C$ be a coalgebra, $A$ an algebra and a right $C$-comodule. Let

$$
B=\left\{b \in A \mid \forall a \in A, \rho_{A}(b a)=b \rho_{A}(a)\right\},
$$

and assume that the canonical right $A$-module, right $C$ comodule map

$$
\text { can }: A \otimes_{B} A \rightarrow A \otimes C, a \otimes a^{\prime} \rightarrow a \rho_{A}\left(a^{\prime}\right)
$$

is bijective. Let $\psi: C \otimes A \rightarrow A \otimes C$ be a $k$-linear map given by

$$
\psi(c \otimes a)=\operatorname{can}\left(\operatorname{can}^{-1}\left(1_{A} \otimes c\right) a\right) .
$$

Then $(A, C, \psi)$ is an entwining structure. The extension $B \subset A$ is called a coalgebra-Galois extension and denoted by $A(B)^{C}$. $(A, C, \psi)$ is the canonical entwining structure associated to $A(B)^{C}$.

Lemma 2.6 Let $(A, C, \psi)$ be a right-right entwining structure. where $\psi$ is invertible, its inverse is $\varphi: A \otimes C \rightarrow C \otimes A$, then $(A, C, \varphi)$ is a left-left entwining structure. i.e.

$$
\begin{gathered}
(a b)_{\varphi} \otimes c^{\varphi}=a_{\varphi} b_{\Phi} \otimes c^{\Phi \varphi}, \\
\left(1_{A}\right)_{\varphi} \otimes c^{\varphi}=1_{A} \otimes c, \\
a_{\varphi} \otimes \Delta_{C}\left(c^{\varphi}\right)=a_{\varphi \Phi} \otimes c_{(1)}^{\varphi} \otimes c_{(2)}^{\Phi}, \\
\varepsilon_{C}\left(c^{\varphi}\right) a_{\varphi}=\varepsilon_{C}(c) a,
\end{gathered}
$$

for all $a, b \in A, c \in C$, where

$$
\varphi(c \otimes a)=a_{\varphi} \otimes c^{\varphi}=a_{\Phi} \otimes c^{\Phi} .
$$

Definition 2.7 Let $(A, C, \psi)$ be an entwining structure. An $(A, C, \psi)$-entwined module is a $k$-module $M$ with a right $A$-action and a right $C$-coaction such that for all $a \in A, m \in M$,

$$
\rho_{M}(m \cdot a)=m_{\langle 0\rangle} \cdot a_{\psi} \otimes m_{\langle 1\rangle}^{\psi} .
$$

A module morphism of entwining structure $(A, C, \psi)$ is a right $A$-module map and a right $C$-comodule map. Generally, we denote the module category of $(A, C, \psi)$ by $\mathcal{M}(\psi)_{\mathrm{A}}^{C}$. Modules associated to the entwining structure in Example 2.4 are Doi-Hopf module. But entwined modules associated to the entwining structure in Example 2.5 do not seem to be of Doi-Hopf type.

Let $F^{C}: \mathcal{M}(\psi)_{A}^{C} \rightarrow \mathcal{M}_{A}$ be the forgetful functor which forgets the $C$-coaction and

$$
\cdot \otimes C: \mathcal{M}_{A} \rightarrow \mathcal{M}(\psi)_{A}^{C}, M \rightarrow M \otimes C
$$

its right adjoint, where the structure maps on $M \otimes C$ are given by

$$
\begin{gathered}
(m \otimes c) \cdot a=m a_{\psi} \otimes c^{\psi}, \\
\rho_{M \otimes C}(m \otimes c)=m \otimes c_{(1)} \otimes c_{(2)},
\end{gathered}
$$

for any $a \in A, c \in C, m \in M$. The unit of the adjoint pair $\left(F^{C}, \otimes C\right)$ is

$$
\rho: 1_{\mathcal{M}(\psi)_{A}^{C}} \rightarrow(\cdot \otimes C) \cdot F^{C}
$$

the $C$-coaction $\rho_{M}: M \rightarrow M \otimes C$, therefor $\rho_{M}$ is $A$ linear and $C$-colinear and can be viewed as a natural transformation between the functor $1_{\mathcal{M}(\psi)_{A}^{C}}$ and $(\cdot \otimes C) \cdot F^{C}$. $A$ is a right $A$-module, so $A \otimes C$ and $A \otimes C \otimes C$ are entwining module via:

$$
\begin{gathered}
(b \otimes c) \cdot a=b a_{\psi} \otimes c^{\psi}, \\
\rho_{A \otimes C}(b \otimes c)=b \otimes c_{(1)} \otimes c_{(2)}, \\
(b \otimes c \otimes d) \cdot a=b a_{\psi \psi \psi} \otimes c^{\Psi} \otimes d^{\psi}, \\
\rho_{A \otimes C \otimes C}(b \otimes c \otimes d)=b \otimes c \otimes d_{(1)} \otimes d_{(2)},
\end{gathered}
$$

for any $a, b \in A, c, d \in C$.

Let $G_{A}: \mathcal{M}(\psi)_{A}^{C} \rightarrow \mathcal{M}^{C}$ be the other forgetful functor which forgets the $A$-action and

$$
\cdot \otimes A: \mathcal{M}^{C} \rightarrow \mathcal{M}(\psi)_{A}^{C}, \quad N \rightarrow N \otimes A
$$

its left adjoint, where for $N \in \mathcal{M}(\psi)_{A}^{C}, N \otimes A$ via the structures

$$
\begin{gathered}
\rho_{N \otimes A}(n \otimes a)=n_{\langle 0\rangle} \otimes a_{\psi} \otimes n_{\langle 1\rangle}^{\psi}, \\
(n \otimes a) b=n \otimes a b,
\end{gathered}
$$

for any $a, b \in A, n \in N$. The unit and counit of the adjoint pair $\left(\cdot \otimes A, G_{A}\right)$ are

$$
\begin{gathered}
\alpha:(\cdot \otimes A) G_{A} \rightarrow 1_{\mathcal{M}(\psi)_{A}^{C}}, \beta: 1_{\mathcal{M}^{C}} \rightarrow G_{A}(\cdot \otimes A), \\
\alpha_{M}: M \otimes A \rightarrow A, \alpha_{M}(m \otimes a)=m a, \\
\beta_{N}: N \rightarrow N \otimes A, \beta_{N}(n)=n \otimes 1 .
\end{gathered}
$$

In particular, $C \otimes A, C \otimes A \otimes A \in \mathcal{M}(\psi)_{A}^{C}$.

\section{The Integrals of the Entwining Structure}

In this section, we first present a point of view which is 
essential for the rest of paper: the existence of an integral on a Hopf algebra is a necessary and sufficient criterion for constructing a natural transformation between two functors.

Lemma 3.1 [6] Let $H$ be a finite dimension Hopf algebra over $k, H^{*}$ is its dual. There exists a right integral $\gamma \in H^{*}$ on $H$ such that $\gamma h^{*}=\left\langle h^{*}, 1_{H}\right\rangle \gamma$ for all $h^{*} \in H^{*}$ if and only if $\gamma: H \rightarrow k$ is right $H$ comodule map, where $k$ has the trivial right $H$-comoule structure.

Doi [11] generalizes this result as follows.

Definition 3.2 Let $A$ be a $H$-comodule algebra. A map $\gamma: H \rightarrow A$ is called an integral if $\gamma$ is right $H$-linear. $\gamma$ is called a total integral if additionally $\gamma\left(1_{H}\right)=1_{A}$.

The criterion for the existence of a total integral is given by the theorem following.

Theorem 3.3 [11] Let $A$ be a right $H$-comodule algebra. The following are equivalent

1) There exists a total integral $\gamma: H \rightarrow A$;

2) Any Hopf module $M \in \mathcal{M}^{H}$ is injective as right $H$-comodule. i.e. the right $H$-coaction $\rho: M \rightarrow M \otimes H$ splits in the category $\mathcal{M}^{H}$;

3) $\rho_{A}: A \rightarrow A \otimes H$ splits in the category $\mathcal{M}^{H}$.

The theorem of Doi can be restated as follows.

Theorem 3.4 [6] Let $A$ be a right $H$-comodule algebra. The following are equivalent

1) There exists a total integral $\gamma: H \rightarrow A$

2) There exists a natural transformation

$\lambda: F_{A} \circ(\cdot \otimes H) \circ F^{H} \rightarrow F_{A} \circ 1_{\mathcal{M}_{A}^{H}}$ that splits

$\rho: F_{A} \circ 1_{\mathcal{M}_{A}^{H}} \rightarrow F_{A} \circ(\cdot \otimes H) \circ F^{H} ;$

3) $\rho_{A}: A \rightarrow A \otimes H$ splits in the category $\mathcal{M}^{H}$.

Remark 3.5 1) The above theorem is still valid leaving aside the normalizing condition $\gamma\left(1_{H}\right)=1_{A}$. More exactly, there exists an integral $\gamma: H \rightarrow A$ if and only if there exists $\lambda: F_{A} \circ(\cdot \otimes H) \circ F^{H} \rightarrow F_{A} \circ 1_{\mathcal{M}_{A}^{H}}$. In particular, if $A=k$, we obtain that there exists a right integral $\gamma: H \rightarrow k$ on $H$ if and only if there exists a natural transformation $\lambda:(\cdot \otimes H) \circ F^{H} \rightarrow 1_{\mathcal{M}^{H}}$. Furthermore, $\quad \gamma\left(1_{H}\right)=1_{k} \quad$ if and only if $\lambda$ splits $\rho: 1_{\mathcal{M}^{H}} \rightarrow(\cdot \otimes H) \circ F^{H}$. This is equivalent to the fact that the forgetful functor $F^{H}: \mathcal{M}^{H} \rightarrow \mathcal{M}_{k}$ is separable.

2) Let $A$ be a right $H$-comodule algebra. The version of Theorem 3.4 for the category $\mathcal{M}_{A}^{H}$ is still true. In this case the $H$-colinear split of

$\rho_{M}: M \rightarrow M \otimes H$ associated to a right total integral $\gamma: H \rightarrow A$ is given by the formula

$$
\begin{gathered}
\lambda_{M}^{\prime}: M \otimes H \rightarrow M, \\
\lambda_{M}^{\prime}(m \otimes h)=m_{\langle 0\rangle} \gamma\left(S\left(m_{\langle 1\rangle}\right) h\right) .
\end{gathered}
$$

We will now give the definition of integral of entwine- ing structure arising from Theorem 2.4 and the definition of integral of Doi-Koppinen structure in [6].

Definition 3.6 Let $(A, C, \psi)$ be an entwining structure. A $k$-linear map $\gamma: C \rightarrow \operatorname{Hom}(C, A)$ is called an integral of $(A, C, \psi)$ if

$$
\gamma\left(c_{(1)}\right)(d) \otimes c_{(2)}=\gamma(c)\left(d_{(2)}\right)_{\psi} \otimes d_{(1)}^{\psi},
$$

for all $c, d \in C$. An integral $\gamma: C \rightarrow \operatorname{Hom}(C, A)$ is called normalized if for all $c \in C$

$$
\gamma\left(c_{(2)}\right) c_{(1)}=\varepsilon(c) 1_{A}
$$

Remark 3.7 If Doi-Koppinen structure $(H, A, C)$ in Example 2.4 takes place of the entwining structure in the above definition and obviously entwining structure map is

$$
\psi: C \otimes A \rightarrow A \otimes C, c \otimes a \rightarrow a_{\langle 0\rangle} \otimes c \cdot a_{\langle 1\rangle} .
$$

Definition 3.6 is just the definition of integral of DoiKoppinen structure.

We shall now prove that the existence of an integral $\gamma: C \rightarrow \operatorname{Hom}(C, A)$ permits the deformation of $k-$ linear map between two entwined modules until it becomes a $C$-colinear map.

Proposition 3.8 Let $(A, C, \psi)$ be an entwining structure. $M \in \mathcal{M}(\psi)_{A}^{C}, N \in \mathcal{M}^{C}$ and $u: N \rightarrow M$ a $k$ linear map. Suppose that there exists $\gamma: C \rightarrow \operatorname{Hom}(C, A)$. Then

1) For all $n \in N$, the map

$\tilde{u}: N \rightarrow M, \tilde{u}(n)=u\left(n_{\langle 0\rangle}\right)_{\langle 0\rangle} \gamma\left(n_{\langle 1\rangle}\right)\left(u\left(n_{\langle 0\rangle}\right)_{\langle 1\rangle}\right)$ is right $C$-colinear;

2) If $\gamma: C \rightarrow \operatorname{Hom}(C, A)$ is a normalized integral and $f: M \rightarrow N$ is a morphism in $\mathcal{M}(\psi)_{A}^{C}$ which is a $k$-split injection (resp. a $k$-split surjection), then $f$ has a $C$-colinear retraction(resp. a section).

Proof. 1) For $n \in N$, we have

$$
\begin{aligned}
& \rho_{M}(\tilde{u}(n)) \\
& =\rho_{M}\left(u\left(n_{\langle 0\rangle}\right)_{\langle 0\rangle} \gamma\left(n_{\langle 1\rangle}\right)\left(u\left(n_{\langle 0\rangle}\right)_{\langle 1\rangle}\right)\right) \\
& =\left(u\left(n_{\langle 0\rangle}\right)_{\langle 0\rangle} \gamma\left(n_{\langle 1\rangle}\right)\left(u\left(n_{\langle 0\rangle}\right)_{\langle 1\rangle(2)}\right)\right)_{\psi} \otimes\left(u\left(n_{\langle 0\rangle}\right)_{\langle 1\rangle(1)}\right)^{\psi} \\
& =u\left(n_{\langle 0\rangle}\right)_{\langle 0\rangle} \gamma\left(n_{\langle 1\rangle(1)}\right) u\left(n_{\langle 0\rangle}\right)_{\langle 1\rangle} \otimes n_{\langle 1\rangle(2)} \\
& =u\left(n_{\langle 0\rangle\langle 0\rangle}\right)_{\langle 0\rangle} \gamma\left(n_{\langle 0\rangle\langle 1\rangle}\right) u\left(n_{\langle 0\rangle\langle 0\rangle}\right)_{\langle 1\rangle} \otimes n_{\langle 1\rangle} \\
& =\tilde{u}\left(n_{\langle 0\rangle}\right) \otimes n_{\langle 1\rangle}=(\tilde{u} \otimes i d) \rho_{N}(n) .
\end{aligned}
$$

2) Let $u: N \rightarrow M$ be a $k$-linear retraction(resp. section) of $f$. Then $\tilde{u}: N \rightarrow M$ is a right $C$-colinear retraction(resp. a section) of $f$. Then, for $m \in M$ 


$$
\begin{aligned}
& (\tilde{u} \circ f)(m) \\
& =u\left(f(m)_{\langle 0\rangle}\right)_{\langle 0\rangle} \gamma\left(f(m)_{\langle 1\rangle}\right) u\left(f(m)_{\langle 0\rangle}\right)_{\langle 1\rangle} \\
& =u\left(f(m)_{\langle 0\rangle}\right)_{\langle 0\rangle} \gamma\left(m_{\langle 1\rangle}\right) u\left(f(m)_{\langle 0\rangle}\right)_{\langle 1\rangle} \\
& =m_{\langle 0\rangle} \gamma\left(m_{\langle 2\rangle}\right)\left(m_{\langle 1\rangle}\right)=m,
\end{aligned}
$$

hence $\tilde{u}$ is a right $C$-colinear retraction of $f$.

On the other hand, if $u: N \rightarrow M$ is a section of $f$, then for $n \in N$

$$
\begin{aligned}
& (f \circ \tilde{u})(n) \\
& =f\left(u\left(n_{\langle 0\rangle}\right)_{\langle 0\rangle} \gamma\left(n_{\langle 1\rangle}\right)\left(u\left(n_{\langle 0\rangle}\right)_{\langle 1\rangle}\right)\right) \\
& =\left(f\left(u\left(n_{\langle 0\rangle}\right)\right)\right)_{\langle 0\rangle} \gamma\left(n_{\langle 1\rangle}\right)\left(f\left(u\left(n_{\langle 0\rangle}\right)\right)\right)_{\langle 1\rangle} \\
& =n_{\langle 0\rangle} \gamma\left(n_{\langle 2\rangle}\right)\left(n_{\langle 1\rangle}\right)=n .
\end{aligned}
$$

i.e. $\tilde{u}$ is a right $C$-colinear section of $f$.

Definition 3.9 [11] A right $C$-comodule $M$ is called injective, if for any $k$-split monomorhism $i: U \rightarrow V$ in $\mathcal{M}^{C}$ and for any $C$-colinear map $f: U \rightarrow M$, there exists $C$-colinear map $g: V \rightarrow M$ such that $g \circ i=f$.

Lemma 3.10 [11] A right $C$-comodule $M$ is injective, if $\rho_{M}: M \rightarrow M \otimes C$ splits in $\mathcal{M}^{C}$, i.e. there exists a $C$-colinear map $\lambda_{M}: M \otimes C \rightarrow M$ such that $\lambda_{M} \circ \rho_{M}=i d_{M}$.

We will prove now the version of Theorem 3.4 for entwining modules which have inverse entwining structure map. Parts of the following theorem are closely related to the ideas presented in [6].

Theorem 3.11 Let $(A, C, \psi)$ be an entwining structure, where $\psi$ is invertible, its inverse is $\varphi$. The following statements are equivalent:

1) There exists a normalized integral $\gamma: C \rightarrow \operatorname{Hom}(C, A)$;

2) The natural transformation

$\rho: F_{A} \circ 1_{\mathcal{M}(\psi)^{C}} \rightarrow F_{A} \circ(\cdot \otimes C) \circ F^{C}$ splits;

3) The right $C$-coaction on $A \otimes C$, $\rho_{A \otimes C}^{r}: A \otimes C \rightarrow A \otimes C \otimes C$ splits in ${ }^{C} \mathcal{M}^{C}$.

Consequently, if one of the equivalent conditions holds, any entwined module is injective as a right $C$ comodule.

Proof. $(1) \Rightarrow(2)$ Let $\gamma: C \rightarrow \operatorname{Hom}(C, A)$ be a normalized integral. We have to construct a natural transformation $\lambda$ that splits $\rho$. Let $M \in \mathcal{M}(\psi)_{A}^{C}$ and $u_{M}: M \otimes C \rightarrow M, \quad u_{M}(m \otimes c)=\varepsilon(c) m$ be the $k$-linear retraction of $\rho_{M}: M \rightarrow M \otimes C$ given by

$u_{M}(m \otimes c)=\varepsilon(c) m$, for all $c \in C, m \in M$. We define $\lambda_{M}=\tilde{u}_{M}$, i.e. for all $c \in C, m \in M$,

$$
\lambda_{M}=\lambda_{M}(\gamma): M \otimes C \rightarrow M,
$$

$$
\lambda_{M}(m \otimes c)=m_{\langle 0\rangle} \gamma(c)\left(m_{\langle 1\rangle}\right) .
$$

It follows from Proposition 3.8 that the map $\lambda_{M}$ is a right $C$-colinear retraction of $\rho_{M}$.

It remains to prove that $\lambda=\lambda(\gamma)$ is a natural transformation. Let $f: M \rightarrow N$ be a morphism in $\mathcal{M}(\psi)_{A}^{C}$. We have to prove that

$$
f \circ \lambda_{M}=\lambda_{N} \circ\left(f \otimes i d_{C}\right) .
$$

Since $f$ is right $A$-linear, we have

$$
\begin{aligned}
& \left(f \circ \lambda_{M}\right)(m \otimes c) \\
= & f\left(m_{\langle 0\rangle} \gamma(c)\left(m_{\langle\backslash\rangle}\right)\right) \\
= & f\left(m_{\langle 0\rangle}\right) \gamma(c)\left(m_{\langle 1\rangle}\right), \\
( & \left.\lambda_{N} \circ\left(f \otimes i d_{C}\right)\right)(m \otimes c) \\
= & \lambda_{N}(c \otimes f(m)) \\
= & f(m)_{\langle 0\rangle} \gamma(c)\left(f(m)_{\langle 1\rangle}\right) \\
= & f\left(m_{\langle 0\rangle}\right) \gamma(c)\left(m_{\langle\backslash\rangle}\right) .
\end{aligned}
$$

i.e. $\lambda$ is a natural transformation that splits $\rho$.

$(2) \Rightarrow(3)$ Assume that for any $M \in \mathcal{M}(\psi)_{A}^{C}$, the $C$-coaction splits in $\mathcal{M}^{C}$. In particular,

$\rho_{A \otimes C}^{r}: A \otimes C \rightarrow A \otimes C \otimes C$ splits in $\mathcal{M}^{C}$. Let

$\lambda=\lambda_{A \otimes C}: A \otimes C \otimes C \rightarrow A \otimes C$ be a right $C$-colinear retraction of $\rho_{A \otimes C}^{r}$. Using the naturality of $\lambda_{A \otimes C}$, we will prove that $\lambda_{A \otimes C}$ is also left $C$-colinear, where $A \otimes C$ and $A \otimes C \otimes C$ are left $C$-comodules via:

$$
\begin{aligned}
& \rho^{l}(a \otimes c)=c_{(1)}^{\varphi} \otimes a_{\varphi} \otimes c_{(2)}, \\
& \rho^{l}(a \otimes c \otimes d)=c_{(1)}^{\varphi} \otimes a_{\varphi} \otimes c_{(2)} \otimes d .
\end{aligned}
$$

Let $V$ be a $k$-module and $M \in \mathcal{M}(\psi)_{A}^{C}$. Then $V \otimes M \in \mathcal{M}(\psi)_{A}^{C}$ via the structures arising from the ones of $M$ as follows

$$
(v \otimes m)=v \otimes m a, \quad \rho_{V \otimes M}=i d_{V} \otimes \rho_{M} .
$$

Using the naturality of $\lambda$, we shall prove that

$$
\lambda_{V \otimes M}=i d_{V} \otimes \lambda_{M} .
$$

Let $v \in V$ and $g_{V}: M \rightarrow V \otimes M, g_{V}(m)=v \otimes m$. From the naturality of $\lambda$ we obtain that

$$
g_{V} \circ \lambda_{M}=\lambda_{V \otimes M} \circ\left(g_{V} \otimes i d_{C}\right) .
$$

Hence

$$
\begin{aligned}
& \lambda_{V \otimes M}(v \otimes m \otimes c) \\
& =g_{V} \circ \lambda_{M}(m \otimes c)=v \otimes \lambda_{M}(m \otimes c) \\
& =\left(i d_{V} \otimes \lambda_{M}\right)(v \otimes m \otimes c) .
\end{aligned}
$$

In particular, let $M=A \otimes C, V=C$, then 
$C \otimes A \otimes C \in \mathcal{M}(\psi)_{A}^{C}$ via the structures arising from the ones of $A \otimes C$, i.e. for all $a, b \in A, c, d \in C$,

$$
\begin{gathered}
(c \otimes b \otimes d) \cdot a=c \otimes b a_{\psi} \otimes d^{\psi}, \\
\rho_{C \otimes A \otimes C}^{r}(c \otimes b \otimes d)=c \otimes b \otimes d_{(1)} \otimes d_{(2)} .
\end{gathered}
$$

With these structures the map

$$
\begin{gathered}
f=\rho_{A \otimes C}^{l}: A \otimes C \rightarrow C \otimes A \otimes C, \\
f(a \otimes C)=c_{(1)}^{\varphi} \otimes a_{\varphi} \otimes c_{(2)}
\end{gathered}
$$

is a morphism in $\mathcal{M}(\psi)_{A}^{C}$. From the naturality of $\lambda$, the following diagram is commutative.

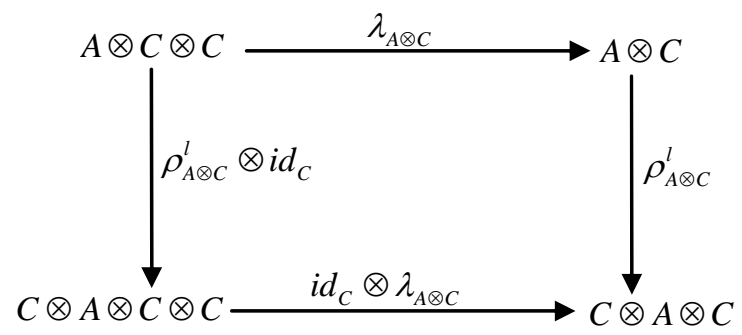

i.e. $\lambda=\lambda_{A \otimes C}$ is also left $C$-colinear.

(3) $\Rightarrow(1)$ The right $C$-coaction

$\rho_{A \otimes C}^{r}: A \otimes C \rightarrow A \otimes C \otimes C$ is a $C$-bicomodule map. Let $\lambda=\lambda_{A \otimes C}: A \otimes C \otimes C \rightarrow A \otimes C$ be a split of $\rho_{A \otimes C}^{r}$ in ${ }^{C} \mathcal{M}^{C}$. In particular, for all $a \in A, c \in C$,

$$
\lambda\left(a \otimes c_{(1)} \otimes c_{(2)}\right)=a \otimes c .
$$

For all $c, d \in C$, define

$$
\begin{gathered}
\gamma: C \rightarrow \operatorname{Hom}(C, A), \\
\gamma(c)(d)=\left(i d_{A} \otimes \epsilon\right) \lambda\left(1_{A} \otimes d \otimes c\right) .
\end{gathered}
$$

We will prove that $\gamma$ is a normalized integral.

Because the right $C$-coaction $\rho_{A \otimes C}^{r}$ is a $C$-bimodule map, on the one hand

$$
\begin{aligned}
& \gamma\left(c_{(1)}\right)(d) \otimes c_{(2)} \\
= & \left(i d_{A} \otimes \epsilon\right) \lambda\left(1_{A} \otimes d \otimes c_{(1)}\right) \otimes c_{(2)} \\
= & \left(i d_{A} \otimes \epsilon \otimes i d_{C}\right)\left(\lambda \otimes i d_{C}\right) \\
& \rho_{A \otimes C \otimes C}^{r}\left(1_{A} \otimes d \otimes C\right) \\
= & \left(i d_{A} \otimes \epsilon \otimes i d_{C}\right) \rho_{A \otimes C}^{r} \lambda\left(1_{A} \otimes d \otimes c\right) \\
= & \lambda\left(1_{A} \otimes d \otimes c\right) ;
\end{aligned}
$$

on the other hand,

$$
\begin{aligned}
& \gamma(c)\left(d_{(2)}\right)_{\psi} \otimes d_{(1)}^{\psi} \\
& =\psi\left(d_{(1)} \otimes \gamma(c)\left(d_{(2)}\right)\right) \\
& =\psi\left(d_{(1)} \otimes\left(i d_{A} \otimes \varepsilon\right) \lambda\left(1_{A} \otimes d_{(2)} \otimes c\right)\right) .
\end{aligned}
$$

We adopt the temporary notion $\lambda\left(1_{A} \otimes d \otimes c\right)=\sum_{i} a_{i} \otimes c_{i} \in A \otimes C$, then

$$
\begin{aligned}
& \rho_{A \otimes C}^{l} \lambda_{A \otimes C}\left(1_{A} \otimes d \otimes C\right) \\
&= \rho_{A \otimes C}^{l}\left(\sum_{i} a_{i} \otimes c_{i}\right)=\sum_{i} c_{i(1)}^{\varphi} \otimes a_{i \varphi} \otimes c_{i(2)}, \\
&\left(i d_{C} \otimes \lambda_{A \otimes C}\right) \rho_{A \otimes C \otimes C}^{l}\left(1_{A} \otimes d \otimes C\right) \\
&=\left(i d_{C} \otimes \lambda_{A \otimes C}\right)\left(d_{(1)} \otimes 1_{A} \otimes d_{(2)} \otimes c\right) \\
&=d_{(1)} \otimes \lambda\left(1_{A} \otimes d_{(2)} \otimes c\right),
\end{aligned}
$$

For $\lambda_{A \otimes C}$ is left $C$-colinear,

$$
\sum d_{(1)} \otimes \lambda\left(1_{A} \otimes d_{(2)} \otimes c\right)=\sum_{i} c_{i(1)}^{\varphi} \otimes a_{i \varphi} \otimes c_{i(2)} .
$$

Hence

$$
\sum d_{(1)} \otimes\left(i d_{A} \otimes \epsilon\right) \lambda\left(1_{A} \otimes d_{(2)} \otimes c\right)=\sum_{i} c_{i}^{\varphi} \otimes a_{i \varphi},
$$

i.e.

$$
\sum \psi\left(d_{(1)} \otimes\left(i d_{A} \otimes \epsilon\right) \lambda\left(1_{A} \otimes d_{(2)} \otimes c\right)\right)=\sum_{i} a_{i} \otimes c_{i} .
$$

We have proved that $\gamma$ is a normalized integral of $(A, C, \psi)$ at last.

Leaving aside the normalizing condition, we obtain the following corollary.

Corollary 3.12 Let $(A, C, \psi)$ be an entwining structure, where $\psi$ is invertible. The following statements are equivalent

1) There exists an integral $\gamma: C \rightarrow \operatorname{Hom}(C, A)$ of $(A, C, \psi)$;

2) There exists $\lambda: F_{A} \circ(\cdot \otimes C) \circ F^{C} \rightarrow F_{A} \circ 1_{\mathcal{M}(\psi)_{A}^{C}}$ a natural transformation;

3) There exists $\lambda^{\prime}: A \otimes C \otimes C \rightarrow A \otimes C$ a $C$-bicomodule map.

If the entwining structure map is invertible, the object $A \otimes C$ take an important role in $\mathcal{M}(\psi)_{A}^{C}$. We shall prove the main application of the existence of a normalized integral in this paper.

Theorem 3.13 Let $(A, C, \psi)$ be an entwining structure, where $\psi$ is invertible, its inverse is $\varphi$. Suppose there exists a normalized integral of $(A, C, \psi)$ $\gamma: C \rightarrow \operatorname{Hom}(C, A)$, for any $M \in \mathcal{M}(\psi)_{A}^{C}$, the map

$$
f: M \otimes A \otimes C \rightarrow M,
$$

$$
f(m \otimes a \otimes c)=m_{\langle 0\rangle} \gamma\left(c^{\varphi}\right)\left(m_{\langle 1\rangle}\right) a_{\varphi}
$$

is a $k$-split epimorphism in $\mathcal{M}(\psi)_{A}^{C}$. In particular, $A \otimes C$ is a generator in the category $\mathcal{M}(\psi)_{A}^{C}$.

Proof. $M \otimes A \otimes C$ is viewed as an object in $\mathcal{M}(\psi)_{A}^{C}$, with the structures arising from the ones of $A \otimes C$, i.e. 


$$
\begin{gathered}
(m \otimes b \otimes c) \cdot a=m \otimes b a_{\psi} \otimes c^{\psi}, \\
\rho(m \otimes b \otimes c)=m \otimes b \otimes c_{(1)} \otimes c_{(2)} .
\end{gathered}
$$

for all $a, b \in A, c \in C, m \in M$. First we shall prove that $f$ is a $k$-split surjection. Let

$$
g: M \rightarrow M \otimes A \otimes C, \quad g(m)=m_{\langle 0\rangle} \otimes 1_{A} \otimes m_{\langle 1\rangle},
$$

for all $m \in M$. Then $g$ is right $C$-colinear. For $m \in M$, we have

$$
\begin{aligned}
(f \circ g)(m) & =f\left(m_{\langle 0\rangle} \otimes 1_{A} \otimes m_{\langle 1\rangle}\right) \\
& =m_{\langle 0\rangle\langle 0\rangle} \gamma\left(m_{\langle 1\rangle}\right)\left(m_{\langle 0\rangle\langle 1\rangle}\right) \\
& =m_{\langle 0\rangle} \gamma\left(m_{\langle 2\rangle}\right)\left(m_{\langle 1\rangle}\right) \\
& =m_{\langle 0\rangle} \epsilon\left(m_{\langle 1\rangle}\right)=m,
\end{aligned}
$$

i.e. $g$ is a right $c$-colinear section of $f$. For $a, b \in A, c \in C, m \in M$, we have

$$
\begin{aligned}
& f((m \otimes b \otimes c) \cdot a) \\
& =f\left(m \otimes b a_{\psi} \otimes c^{\psi}\right)=m_{\langle 0\rangle} \gamma\left(c^{\psi \Phi \varphi}\right)\left(m_{\langle 1\rangle}\right) b_{\varphi} a_{\psi \Phi} \\
& =m_{\langle 0\rangle} \gamma\left(c^{\varphi}\right)\left(m_{\langle 1\rangle}\right) b_{\varphi} a=f(m \otimes b \otimes c) \cdot a,
\end{aligned}
$$

i.e. $f$ is right $A$-linear. It remains to prove that $f$ is also right $C$-colinear. In fact,

$$
\begin{gathered}
\left(f \otimes i d_{C}\right) \rho_{M \otimes A \otimes C}(m \otimes b \otimes c) \\
=\left(f \otimes i d_{C}\right)\left(m \otimes b \otimes c_{(1)} \otimes c_{(2)}\right) \\
=f\left(m \otimes b \otimes c_{(1)}\right) \otimes c_{(2)} \\
=m_{\langle 0\rangle} \gamma\left(c_{(1)}^{\varphi}\right)\left(m_{\langle 1\rangle}\right) b_{\varphi} \otimes c_{(2)}, \\
\rho_{M} f(m \otimes b \otimes c) \\
=\rho_{M}\left(m_{\langle 0\rangle} \gamma\left(c^{\varphi}\right)\left(m_{\langle 1\rangle}\right)\left(b_{\varphi}\right)\right. \\
=m_{\langle 0\rangle\langle 0\rangle}\left(\gamma\left(c^{\varphi}\right)\left(m_{\langle 1\rangle}\right) b_{\varphi}\right)_{\psi} \otimes\left(m_{\langle 0\rangle\langle 1\rangle}\right)^{\psi} \\
=m_{\langle 0\rangle}\left(\gamma\left(c^{\varphi}\right)\left(m_{\langle 2\rangle}\right) b_{\varphi}\right)_{\psi} \otimes m_{\langle 1\rangle}^{\psi} \\
=m_{\langle 0\rangle}\left(\gamma\left(c^{\varphi}\right)\left(m_{\langle 2\rangle}\right)\right)_{\psi} b_{\varphi \Psi} \otimes m_{\langle 1\rangle}^{\psi \Psi} \\
=m_{\langle 0\rangle} \gamma\left(\left(c^{\varphi}\right)_{(1)}\right)\left(m_{\langle 1\rangle}\right) b_{\varphi \Psi} \otimes\left(\left(c^{\varphi}\right)_{(2)}\right)^{\Psi} \\
=m_{\langle 0\rangle} \gamma\left(c_{(1)}^{\varphi}\right)\left(m_{\langle 1\rangle}\right) b_{\varphi \Psi \Psi} \otimes c_{(2)}^{\Phi \Psi} \\
=m_{\langle 0\rangle} \gamma\left(c_{(1)}^{\varphi}\right)\left(m_{\langle 1\rangle}\right) b_{\varphi} \otimes c_{(2)},
\end{gathered}
$$

i.e. $f$ is right $C$-colinear.

Hence, $f$ is an epimorphism in $\mathcal{M}(\psi)_{A}^{C}$ and has a right $C$-colinear section.
Taking a $k$-free presentation of $M$ in the category of $k$-modules

$$
k^{(I)} \stackrel{\phi}{\longrightarrow} M \rightarrow 0,
$$

we obtain an epimorphism in $\mathcal{M}(\psi)_{A}^{C}$

$$
(A \otimes C)^{(I)} \cong k^{(I)} \otimes A \otimes C \stackrel{g}{\longrightarrow} M \rightarrow 0
$$

where $g=f \circ\left(\pi \otimes I_{A} \otimes I_{C}\right)$. Hence $A \otimes C$ is a generator in $\mathcal{M}(\psi)_{A}^{C}$.

Remark 3.14 An important application of integrals in finite dimension Hopf algebra is Maschke theorem. It finds the condition of finite dimension Hopf algebras to be semisimple. [12-14] have studied the relation between the integral of Doi-Koppinen structure and Maschke theorem. The integrals of entwining structure we study here also have a tend relation with Maschke theorem, the readers can refer to the reference $[3,15]$.

\section{The Cointegrals of Entwining Structure}

Because the entwining structure has the property of selfduality, we will get some dual results of Section 3. In order to give the dual definition of integrals of the entwining structure conveniently, we have the lemma as follows

Lemma 4.1 Let $(A, C, \psi)$ be an entwining structure. The following are equivalent:

1) There exists a normalized integral $\gamma: C \rightarrow \operatorname{Hom}(C, A)$;

2) There exists a $k$-linear map $\theta: C \otimes C \rightarrow A$ such that for all $c, d \in C$.

$$
\begin{gathered}
\theta\left(c \otimes d_{(1)}\right) \otimes d_{(2)}=\theta\left(c_{(2)} \otimes d\right)_{\psi} \otimes c_{(1)}^{\psi}, \\
\theta\left(c_{(1)} \otimes c_{(2)}\right)=\epsilon(c) 1_{A .}
\end{gathered}
$$

Proof. Let $\gamma(d)(c)=\theta(c \otimes d)$. The proof of the lemma is obvious.

Definition 4.2 Let $(A, C, \psi)$ be an entwining structure. A $k$-linear map $\delta: C \rightarrow A \otimes A$ is called an cointegral of $(A, C, \psi)$ if for any

$$
\begin{gathered}
c \in C, \delta(c)=\delta^{1}(c) \otimes \delta^{2}(c) \in A \otimes A, \\
\delta^{1}(c) \otimes \delta^{2}(c) a=a_{\psi} \delta^{1}\left(c^{\psi}\right) \otimes \delta^{2}\left(c^{\psi}\right) .
\end{gathered}
$$

A cointegral $\delta$ is called normalized if

$$
\delta^{1}(c) \delta^{2}(c)=\epsilon(c) 1_{A} .
$$

We shall have the dual results of Proposition 3.8, Theorem 3.11 and Theorem 3.13.

Proposition 4.3 Let $(A, C, \psi)$ be a entwining structure. $\quad M \in \mathcal{M}(\psi)_{A}^{C}, \quad N \in \mathcal{M}_{A}, \quad u: N \rightarrow M$ a $k$-linear map. Suppose that there exists $\delta: C \rightarrow A \otimes A$, then: 
1) For all $n \in N$, the map

$$
\tilde{u}: N \rightarrow M, \tilde{u}=u\left(n_{\langle 0\rangle} \delta^{1}\left(n_{\langle 1\rangle}\right)\right) \delta^{2}\left(n_{\langle 1\rangle}\right)
$$

is right $A$-linear;

2) Let $\delta: C \rightarrow A \otimes A$ is a normalized cointegral and $f: M \rightarrow N$ is a morphism in $\mathcal{M}(\psi)_{\mathrm{A}}^{C}$ which is a $k$-split injection (resp. a $k$-split surjection), then $f$ has a $A$-linear retraction(resp. a section).

Proof. It is just the dual of Proposition 3.8.

Theorem 4.4 Let $(A, C, \psi)$ be an entwining structure, where $\psi$ is invertible, its inverse is $\phi$. The following statements are equivalent:

1) There exists a normalized cointegral $\delta: C \rightarrow A \otimes A$;

2) The natural transformation $\mu: G^{C} \circ(\cdot \otimes A) \circ G_{A} \rightarrow G^{C} \circ 1_{\mathcal{M}(\psi)_{A}^{C}}$ splits;

3) The right $A$-action on $C \otimes A$, $\mu_{C \otimes A}^{r}: C \otimes A \otimes A \rightarrow C \otimes A$ splits in ${ }_{A} \mathcal{M}_{A}$.

Consequently, if one of the equivalent conditions holds, any entwined module is projective as a right $C$-comodule.

Proof. (1) $\Rightarrow(2)$ Let $\delta: C \rightarrow A \otimes A$ be a normalized cointegral. We have to construct a natural transformation $\eta$ that splits $\mu$. Let $M \in \mathcal{M}(\psi)_{A}^{C}$ and for any $m \in M, c \in C, u_{M}: M \rightarrow M \otimes A, u_{M}(m)=m \otimes 1_{A}$ be the $k$-linear section of $\mu_{M}: M \otimes A \rightarrow M$. We define $\eta_{M}=\tilde{u}_{M}$, i.e.

$$
\begin{gathered}
\eta_{M}=\eta_{M}(\delta): M \rightarrow M \otimes A, \\
\eta_{M}(m)=m_{\langle 0\rangle} \delta^{1}\left(m_{\langle 1\rangle}\right) \otimes \delta^{2}\left(m_{\langle 1\rangle}\right)
\end{gathered}
$$

where $m \in M$. It follows from Proposition 4.3 that the map $\eta_{M}$ is a right $A$-linear section of $\mu_{M}$. It remains to prove that $\eta=\eta(\delta)$ is a natural transformation.

Let $f: M \rightarrow N$ be a morphism in $\mathcal{M}(\psi)_{A}^{C}$. We have to prove that

$$
\left(f \otimes i d_{A}\right) \circ \eta_{M}=\eta_{N} \circ f .
$$

For any $m \in M$, using that $f$ is right $C$-colinear, we have

$$
\begin{aligned}
& \left(f \otimes i d_{A}\right) \eta_{M}(m) \\
& =\left(f \otimes i d_{A}\right)\left(m_{\langle 0\rangle} \delta^{1}\left(m_{\langle 1\rangle}\right) \otimes \delta^{2}\left(m_{\langle\backslash\rangle}\right)\right) \\
& =f\left(m_{\langle 0\rangle} \delta^{1}\left(m_{\langle 1\rangle}\right)\right) \otimes \delta^{2}\left(m_{\langle 1\rangle}\right) \\
& =f\left(m_{\langle 0\rangle}\right) \delta^{1}\left(m_{\langle 1\rangle}\right) \otimes \delta^{2}\left(m_{\langle\backslash\rangle}\right), \\
& \eta_{N} f(m) \\
& =f(m)_{\langle 0\rangle} \delta^{1}\left(f(m)_{\langle 1\rangle}\right) \otimes \delta^{2}\left(f(m)_{\langle\backslash\rangle}\right) \\
& =f\left(m_{\langle 0\rangle}\right) \delta^{1}\left(m_{\langle 1\rangle}\right) \otimes \delta^{2}\left(m_{\langle 1\rangle}\right),
\end{aligned}
$$

i.e. $\eta$ is a natural transformation that splits $\mu$.

$(2) \Rightarrow(3)$ Assume that for any $M \in \mathcal{M}(\psi)_{A}^{C}$, right $A$-action splits in $\mathcal{M}_{A}$. In particular,

$\mu_{C \otimes A}: C \otimes A \otimes A \rightarrow C \otimes A$ splits in $\mathcal{M}_{A}$. Let $\eta=\eta_{C \otimes A}: C \otimes A \rightarrow C \otimes A \otimes A$ be a right $A$-linear section of $\mu_{C \otimes A}$. Using the naturality of $\eta_{C \otimes A}$, we will prove that $\eta_{C \otimes A}$ is also left $A$-linear, where $C \otimes A$ and $C \otimes A \otimes A$ are left $A$-modules via:

$$
\begin{gathered}
a \cdot(c \otimes b)=c^{\varphi} \otimes a_{\varphi} b, \\
a \cdot\left(c \otimes b \otimes b^{\prime}\right)=c^{\varphi} \otimes a_{\varphi} b \otimes b^{\prime},
\end{gathered}
$$

where $a, b, b^{\prime} \in A, c \in C$.

First let $V$ be a $k$-module and $M \in \mathcal{M}(\psi)_{A}^{C}$. Then $V \otimes M \in \mathcal{M}(\psi)_{A}^{C}$, via the structures arising from the ones of $M$, i.e.

$$
(v \otimes m) a=v \otimes m a, \rho_{V \otimes M}=i d_{V} \otimes \rho_{M} .
$$

Using the naturality of $\eta$, we prove that

$$
\eta_{V \otimes M}=i d_{V} \otimes \eta_{M} \text {. }
$$

Let $v \in V$ and $g_{v}: M \rightarrow V \otimes M, g_{v}(m)=v \otimes m$. From the naturality of $\eta$ we obtain that

$$
\eta_{V \otimes M} \circ g_{V}=\left(g_{V} \otimes i d_{A}\right) \circ \eta_{M} .
$$

Hence

$$
\begin{aligned}
& \eta_{V \otimes M}(v \otimes m) \\
& =\eta_{V \otimes M} \circ g_{V}(m)=\left(g_{V} \otimes i d_{A}\right) \circ \eta_{M}(m) \\
& =v \otimes \eta_{M}(m)=\left(i d_{V} \otimes \eta_{M}\right)(m) .
\end{aligned}
$$

In particular, let $M=C \otimes A, V=A$, then $A \otimes C \otimes A \in \mathcal{M}(\psi)_{A}^{C}$ via the structures arising from the ones of

$$
\begin{gathered}
\left(b^{\prime} \otimes c \otimes b\right) \cdot a=b^{\prime} \otimes c \otimes b a, \\
\rho_{A \otimes C \otimes A}^{r}\left(b^{\prime} \otimes c \otimes b\right)=b^{\prime} \otimes c_{(1)} \otimes b_{\psi} \otimes c_{(2)}^{\psi \prime} .
\end{gathered}
$$

for all $a, b, b^{\prime} \in A, c \in C$. With these structures the map

$$
\begin{gathered}
f=\mu_{C \otimes A}^{l}: A \otimes C \otimes A \rightarrow C \otimes A, \\
a \cdot(c \otimes b)=c^{\varphi} \otimes a_{\varphi} b
\end{gathered}
$$

is a morphism in $\mathcal{M}(\psi)_{A}^{C}$. From the naturality of $\eta$, the following diagram is commutative.

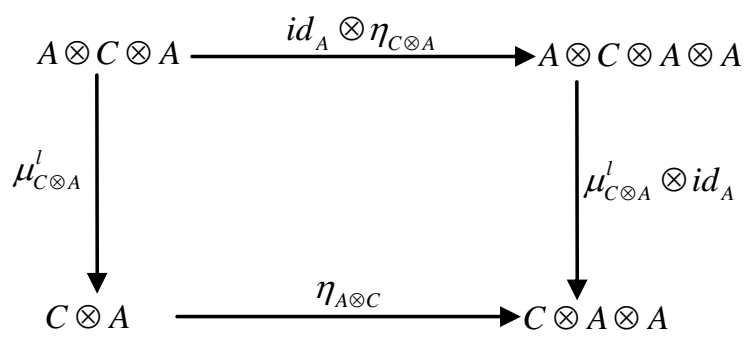


i.e. $\eta=\eta_{C \otimes A}$ is also left $A$-linear.

(3) $\Rightarrow(1)$ : The right $A$-action

$\mu_{C \otimes A}^{r}: C \otimes A \otimes A \rightarrow C \otimes A$ is a $A$-bimodule map. Let $\eta=\eta_{C \otimes A}: C \otimes A \otimes A \rightarrow C \otimes A$ be a split of $\mu_{C \otimes A}^{r}$ in

${ }_{A} \mathcal{M}_{A}$. In particular, for all $a \in A, c \in C$,

$($ id $\otimes m) \eta(c \otimes a)=c \otimes a$.

For all $c \in C$, we define

$$
\delta: C \rightarrow A \otimes A, \quad \delta(c)=\left(\epsilon \otimes i d_{A} \otimes i d_{A}\right) \eta\left(c \otimes 1_{A}\right) .
$$

We will prove that $\delta$ is a normalized cointegral.

$$
\begin{aligned}
& \delta^{1}(c) \delta^{2}(c)=m\left(\epsilon \otimes i d_{A} \otimes i d_{A}\right) \eta\left(c \otimes 1_{A}\right) \\
& =(\epsilon \otimes i d)(i d \otimes m) \eta\left(c \otimes 1_{A}\right)=\epsilon(c) 1_{A} .
\end{aligned}
$$

For any $c \in C, a \in A$, on the one hand

$$
\begin{aligned}
& a_{\psi} \delta^{1}\left(c^{\psi}\right) \otimes \delta^{2}\left(c^{\psi}\right) \\
&=\left(m \otimes i d_{A}\right)\left(i d_{A} \otimes \delta\right) \psi(c \otimes a) \\
&=\left(m \otimes i d_{A}\right)\left(i d_{A} \otimes\left(\epsilon \otimes i d_{A} \otimes i d_{A}\right) \eta\right) \\
&\left(\psi \otimes i d_{A}\right)\left(c \otimes a \otimes 1_{A}\right) \\
&=\left(m \otimes i d_{A}\right)\left(a_{\psi} \otimes\left(\epsilon \otimes i d_{A} \otimes i d_{A}\right) \eta\left(c^{\psi} \otimes 1_{A}\right)\right),
\end{aligned}
$$

Let $\eta\left(c^{\psi} \otimes 1_{A}\right)=\sum c_{j} \otimes a_{j} \otimes b_{j}$, then

$$
a_{\psi} \delta^{1}\left(c^{\psi}\right) \otimes \delta^{2}\left(c^{\psi}\right)^{j}
$$$$
=\left(m \otimes i d_{A}\right)\left(a_{\psi} \otimes\left(\epsilon \otimes i d_{A} \otimes i d_{A}\right)\left(\sum_{j} c_{j} \otimes a_{j} \otimes b_{j}\right)\right)
$$$$
=\sum_{j} \epsilon\left(c_{j}\right) a_{\psi} a_{j} \otimes b_{j} ;
$$

on the other hand,

$$
\delta^{1}(c) \otimes \delta^{2}(c) a
$$$$
=\left(i d_{A} \otimes m\right)\left(\delta \otimes i d_{A}\right)(c \otimes a)
$$$$
=\left(i d_{A} \otimes m\right)\left(\varepsilon \otimes i d_{A} \otimes i d_{A}\right)\left(\eta \otimes i d_{A}\right)\left(c \otimes 1_{A} \otimes a\right)
$$$$
=\left(\epsilon \otimes i d_{A} \otimes i d_{A}\right)\left(i d_{A} \otimes i d_{A} \otimes m\right)\left(\eta \otimes i d_{A}\right)\left(c \otimes 1_{A} \otimes a\right)
$$$$
=\left(\epsilon \otimes i d_{A} \otimes i d_{A}\right) \eta(c \otimes a)
$$$$
=\left(\epsilon \otimes i d_{A} \otimes i d_{A}\right) \eta\left(c^{\psi \varphi} \otimes a^{\psi \varphi}\right)
$$$$
=\left(\epsilon \otimes i d_{A} \otimes i d_{A}\right)\left(a^{\psi} \eta\left(c^{\psi} \otimes 1_{A}\right)\right)
$$$$
=\left(\epsilon \otimes i d_{A} \otimes i d_{A}\right)\left(a^{\psi}\left(\sum_{j} c_{j} \otimes a_{j} \otimes b_{j}\right)\right)
$$$$
=\left(\epsilon \otimes i d_{A} \otimes i d_{A}\right)\left(\sum_{j} c_{j}^{\varphi} \otimes a_{\psi \varphi} a_{j} \otimes b_{j}\right)
$$$$
=\sum_{j} \epsilon\left(c_{j}^{\varphi}\right) a_{\psi \varphi} a_{j} \otimes b_{j}
$$$$
=\sum_{j} \epsilon\left(c_{j}\right) a_{\psi} a_{j} \otimes b_{j},
$$

we have used $\eta$ is a $A$-bimodule map. Hence

$$
\delta^{1}(c) \otimes \delta^{2}(c) a=a_{\psi} \delta^{1}\left(c^{\psi}\right) \otimes \delta^{2}\left(c^{\psi}\right) .
$$

Then we obtained that $\delta$ is a normalized cointegral of $(A, C, \psi)$ finally.

Leaving aside the normalizing condition, we obtain the following corollary

Corollary 4.5 Let $(A, C, \psi)$ be an entwining structure, where $\psi$ is invertible. The following statements are equivalent:

1) There exists a cointegral $\delta: C \rightarrow A \otimes A$;

2) There exists $\eta: G^{C} \circ 1_{\mathcal{M}(\psi)_{A}^{C}} \rightarrow G^{C} \circ(\cdot \otimes A) \circ G_{A}$ a natural transformation;

3) There exists $\eta^{\prime}: C \otimes A \rightarrow C \otimes A \otimes A$ an $A$-bimodule map.

Theorem 4.6 Let $(A, C, \psi)$ be an entwining structure, where $\psi$ is invertible, its inverse is $\phi$. Suppose there exists a normalized cointegral of $(A, C, \psi)$ $\delta: C \rightarrow A \otimes A$, for any $M \in \mathcal{M}(\psi)_{A}^{C}$, the map

$$
\begin{gathered}
f: M \rightarrow M \otimes C \otimes A, \\
f(m)=m_{\langle 0\rangle} \delta^{1}\left(m_{\langle 1\rangle}\right) \otimes m_{\langle 2\rangle}^{\varphi} \otimes \delta^{2}\left(m_{\langle 1\rangle}\right)_{\varphi} .
\end{gathered}
$$

for all $a \in A, c \in C, m \in M$ is a $k$-split monomorphism in $\mathcal{M}(\psi)_{A}^{C}$. In particular, $C \otimes A$ is a cogenerator in the category $\mathcal{M}(\psi)_{\mathrm{A}}^{C}$.

Proof. It is just the dual of Theorem 3.13.

\section{Acknowledgements}

This work was partially supported by the Natural Science Foundation of Henan Province (102300410049).

\section{REFERENCES}

[1] R. G. Larson and M. E. Sweedler, "An Associative Orthogonal Bilinear form for Hopf Algebras," American Journal of Mathematics, Vol. 91, No. 1, 1969, pp. 75-94. doi: $10.2307 / 2373270$

[2] M. E. Sweedler, "Integrals for Hopf Algebra," Annals of Mathematics Second Series, Vol. 89, No. 2, 1969, pp. 323-335. doi: $10.2307 / 1970672$

[3] T. Kerler, "Bridged Links and Tangle Presentations of Cobordism Categories," Advances in Mathematics, Vol. 141, No. 2, 1999, pp. 207-281. doi:10.1006/aima.1998.1772

[4] G. Kuperberg, "Non-Involuntory Hopf Algebras and 3Manifold Invariants," Duke Mathematical Journal, Vol. 84, No. 1, 1996, pp. 83-129. doi:10.1215/S0012-7094-96-08403-3

[5] V. Turaev, "Quantum Invariants of Knots and 3-Manifolds," Walter de Gruyter, Berlin, 1994.

[6] C. Menini and G. Mimitaru, "Integral, Quantum Galois Extensions and the Affineness Criterion for Quantum Yetter-Drinfeld Modules," Journal of Algebra, Vol. 247, No. 2, 2002, pp. 467-508. doi:10.1006/jabr.2001.8899

[7] T. Brezinski and S. Majid, "Coalgebra Bundles," Commu- 
nications in Mathematical Physics, Vol. 191, No. 2, 1998, pp. 467-492. doi:10.1007/s002200050274

[8] T. Brezinski, "On Modules Associated to Coalgebra Galois Extensions," Journal of Algebra, Vol. 215, No. 1, 1999, pp. 290-317. doi:10.1006/jabr.1998.7738

[9] M. E. Sweedler, "Hopf Algebras," Benjamin, New York, 1969.

[10] Y. Doi, "Unifying Hopf Modules," Journal of Algebra, Vol. 153, No. 2, 1992, pp. 373-385. doi:10.1016/0021-8693(92)90160-N

[11] Y. Doi, "Algebras with Total Integrals," Communications in Algebra, Vol. 13, No. 10, 1985, pp. 2137-2159.

[12] S. Caenepeel, G. Militaru and S. Zhu, "Doi-Hopf Modules, Yetter-Drinfel'd Modules and Frobenius Type Properties," Transactions of the American Mathematical Society,
Vol. 349, No. 11, 1997, pp. 4311-4342. doi:10.1090/S0002-9947-97-02004-7

[13] S. Caenepeel, G. Militaru and S. Zhu, "A Maschke Type Theorem for Doi-Hopf Modules and Applications," Journal of Algebra, Vol. 187, No. 2, 1997, pp. 388-412. doi:10.1006/jabr.1996.6794

[14] S. Caenepeel, G. Militaru and S. Zhu, "Separable Functors for the Category of Doi-Hopf Modules, Applications," Advances in Mathematics, Vol. 145, No. 2, 1999, pp. 239-290. doi:10.1006/aima.1998.1817

[15] T. Brzeziński, S. Caenepeel, G. Militaru and S. Zhu, "Frobenius and Maschke Type Theorems for Doi-Hopf Modules and Entwined Modules Revisited: A Unified Approach," In: A. Granja, J. Alonso Hermida and A. Verschoren, Eds., Marcel Dekker, New York, 2001. 